\title{
Temperature-dependent terahertz spectroscopy of inverted-band three-layer InAs/GaSb/InAs quantum well
}

\author{
S. S. Krishtopenko, ${ }^{1,2}$ S. Ruffenach, ${ }^{1}$ F. Gonzalez-Posada, ${ }^{3}$ G. Boissier, ${ }^{3}$ M. Marcinkiewicz, ${ }^{1}$ M. A. Fadeev, ${ }^{1,2}$ \\ A. M. Kadykov, ${ }^{1,2}$ V. V. Rumyantsev, ${ }^{2,4}$ S. V. Morozov, ${ }^{2,4}$ V. I. Gavrilenko, ${ }^{2,4}$ C. Consejo, ${ }^{1}$ W. Desrat, ${ }^{1}$ \\ B. Jouault, ${ }^{1}$ W. Knap, ${ }^{1}$ E. Tournié, ${ }^{3}$ and F. Teppe ${ }^{1, *}$ \\ ${ }^{1}$ Laboratoire Charles Coulomb, Université de Montpellier, Centre National de la Recherche Scientifique, 34095 Montpellier, France \\ ${ }^{2}$ Institute for Physics of Microstructures RAS, GSP-105, 603950 Nizhni Novgorod, Russia \\ ${ }^{3}$ Institut d'Electronique et des Systemes, Université de Montpellier, Centre National de la Recherche Scientifique, 34000 Montpellier, France \\ ${ }^{4}$ Lobachevsky State University of Nizhni Novgorod, prospekt Gagarina 23, Nizhni Novgorod 603950, Russia
}

(Received 27 March 2018; published 22 June 2018)

\begin{abstract}
We report on temperature-dependent terahertz spectroscopy of a three-layer InAs/GaSb/InAs quantum well $(\mathrm{QW})$ with inverted-band structure. The interband optical transitions, measured up to $16 \mathrm{~T}$ at different temperatures by Landau-level magnetospectroscopy, demonstrate the inverted-band structure of the $\mathrm{QW}$. The terahertz photoluminescence at different temperatures allows us to directly extract the optical gap in the vicinity of the $\Gamma$ point of the Brillouin zone. Our results experimentally demonstrate that the gap in the three-layer QWs is temperature independent and exceeds by four times the maximum band gap available in the inverted InAs/GaSb bilayers.
\end{abstract}

DOI: 10.1103/PhysRevB.97.245419

\section{INTRODUCTION}

A two-dimensional topological insulator (2D TI), also known as a quantum spin Hall insulator (QSHI) is a state of matter characterized by an insulating bulk and spin-polarized gapless helical states at the sample edges [1-6]. So far, the helical nature of the edges states in QSHIs has been experimentally demonstrated only in $\mathrm{HgTe} / \mathrm{CdHgTe}$ quantum wells (QWs) [7] and broken-gap InAs/GaSb QW bilayers [8].

In $\mathrm{HgTe}$ QWs, the inverted-band structure responsible for the QSHI state arises due to the strong spin-orbit interaction in the $\mathrm{HgTe}$ layer. The position of the first electronlike $E 1$ and holelike $H 1$ subbands therefore depends on the QW width. The QWs wider than a critical value $d_{c}$ feature subband inversion [9], while the QWs thinner than $d_{c}$ have a noninverted-band ordering and a trivial band insulator (BI) state. The maximum inverted-band gap achievable in classical tensile-strained $\mathrm{HgTe}$ QWs grown on CdTe does not exceed $16 \mathrm{meV}$, but it can reach $55 \mathrm{meV}$ in compressively strained $\mathrm{HgTe}$ QWs [10].

The subband inversion in InAs/GaSb bilayer QWs is caused by specific band-edge alignment of InAs and GaSb semiconductors arising at their interface [11-13]. For thin enough InAs and GaSb layers, the $E 1$ level lies above the holelike $H 1$ level, and the QW has a trivial band ordering. With increasing the layer thickness, an inverted-band ordering may arise, in which the $E 1$ subband becomes lower than the $H 1$ subband. The inherent property of bilayer InAs/GaSb QWs is the lack of inversion symmetry in the growth direction, which leads to the crossing of $E 1$ and $H 1$ and, hence, to the opening of a hybridization band gap at nonzero quasimomentum [14]. The inverted-band gap in QSHIs made of $\mathrm{InAs} / \mathrm{GaSb}$ bilayers is

*frederic.teppe@umontpellier.fr about 3-4 meV only [4], but it can rise up to $20 \mathrm{meV}$ in strained InAs/GaInSb bilayer QWs [15,16].

The inverted band in InAs/GaSb QW bilayers gap opens at a finite value of the quasimomentum $\mathbf{k}$, due to the interaction between $E 1$ and $H 1$ subbands. In HgTe QWs, the inverted-band gap arises in the $\Gamma$ point due to quantum confinement, while the $E 1-H 1$ interaction vanishes at $\mathbf{k}=0$. As shown recently, removing the structure inversion asymmetry by adding a second InAs layer to the InAs/GaSb QW bilayers [see Fig. 1(a)] leads to the crossing of $E 1$ and $H 1$ subbands at $k=0$ for a specific thickness of the layers and to a QSHI state with an inverted-band gap as high as $16 \mathrm{meV}$ for wider QWs [17]. This gap is therefore comparable with the inverted gap in tensile-strained $\mathrm{HgTe}$ QWs. Interestingly, the strained threelayer InAs/GaInSb/InAs QWs are predicted to host QSHI state with a band gap as large as in compressively strained $\mathrm{HgTe}$ QWs [17].

Very recently, a quantum spin Hall effect (QSHE) in $1 T^{\prime}-\mathrm{WTe}_{2}$ monolayers has been observed at temperatures up to $100 \mathrm{~K}$ [18]. Note that experimental observation of QSHE in monolayer systems is challenging, often owing to structural or chemical instabilities [19-21], which also stimulates the search for candidates for the realization of high-temperature QSHI among QW heterostructures. Recent spectroscopic measurements $[22,23]$ performed in the $\mathrm{WTe}_{2}$ monolayers revealed an inverted-band gap of $45 \mathrm{meV}$, which is actually comparable with the gaps in compressively strained HgTe QWs [10] and three-layer InAs/GaInSb/InAs QWs [17].

However, a relatively large inverted gap in the $\mathrm{HgTe}$ QWs is only available at low temperatures, while increasing the temperature leads to a gap closing and a phase transition into a trivial BI state [24-28]. The latter is caused by strong temperature dependence of mutual position of the $\Gamma_{6}$ and $\Gamma_{8}$ bands in bulk HgCdTe crystals [29]. Since temperature does not 

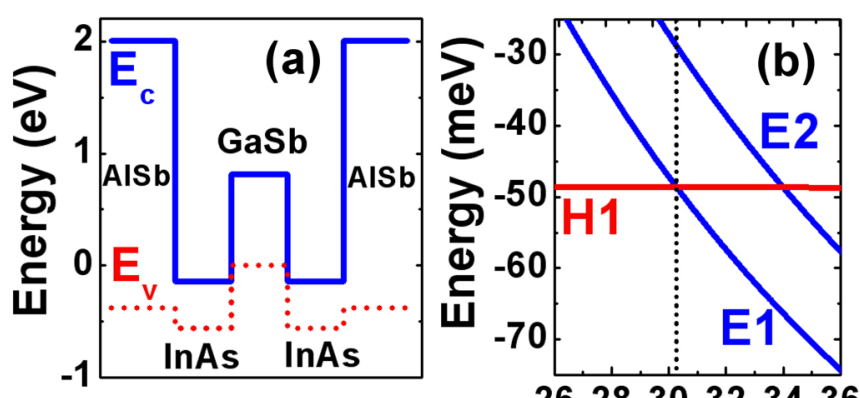

262830323436

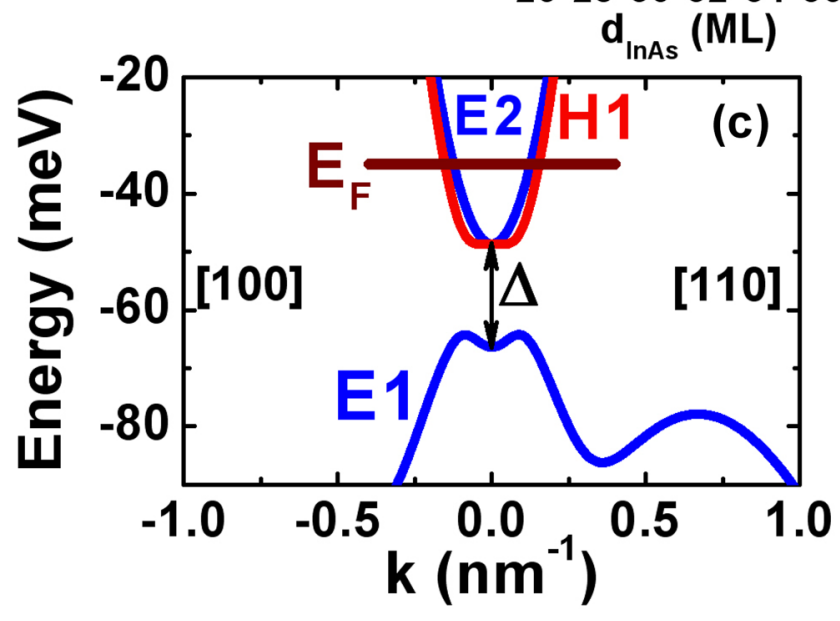

FIG. 1. (a) Band-edge diagram for three-layer InAs/GaSb QWs grown on GaSb buffer. The zero energy is referenced to the valenceband edge of bulk GaSb. Outer AlSb barriers provide an overall confining potential for electronlike and holelike states. (b) Position of electronlike (blue curves) and holelike (red curve) subbands at zero quasimomentum as a function of InAs layer thickness $d_{\text {InAs }}$. The vertical dotted line separates the regions corresponding to the trivial and inverted band ordering. (c) Calculated band structure. Numbers in brackets denote crystal directions. The brown line indicates the position of the Fermi level $E_{F}$ found from magnetotransport measurements.

affect the mutual ordering of the $\Gamma_{6}$ and $\Gamma_{8}$ bands in InSb, InAs, and $\mathrm{GaSb}$ semiconductors, one can expect a weaker band-gap dependence on temperature in the QSHIs based on these III-V semiconductors.

In this work, we address the question of temperature dependence of the band structure in inverted three-layer InAs/GaSb/InAs QWs. We report on temperature-resolved terahertz $(\mathrm{THz})$ spectroscopy of these structures with the layer thicknesses corresponding to the QSHI state. To probe its band structure, we perform Landau-level (LL) magnetospectroscopy in magnetic fields up to $16 \mathrm{~T}$ in a temperature range from $2 \mathrm{~K}$ up to $100 \mathrm{~K}$ (Sec. III). This method also makes it possible to estimate the energy gap between different subbands in the QW by following the characteristic LL transitions from high to low magnetic fields [30-35]. The observed LL optical transitions clearly reveal the inverted band ordering of our sample. To extract the optical gap between the QW subbands, we also performed $\mathrm{THz}$ photoluminescence (PL) measurements [36,37] at zero magnetic field (Sec. IV). The main results of this work are summarized and discussed in Sec. V.

\section{SAMPLE PARAMETERS}

Figure 1(a) schematically shows the band-edge diagram for three-layer InAs/GaSb/InAs QWs confined by outer AlSb barriers grown on (001) GaSb buffer layers. As it is mentioned above, the broken-gap alignment at InAs/GaSb interface leads to the possibility of the band inversion in InAs/GaSb-based QWs [4,11-13] realized at certain thickness of InAs and GaSb layers. The absence of structure inversion asymmetry in the growth direction leads to the crossing of electronlike and holelike subbands in the three-layer QWs at zero quasimomentum.

Figure 1(b) presents the position of electronlike $(E 1, E 2)$ and holelike $(H 1)$ subbands at $\mathbf{k}=0$ as a function of InAs-layer thickness $d_{\text {InAs }}$ for GaSb-layer thickness of $d_{\mathrm{GaSb}}=14 \mathrm{ML}$, where 1 monolayer (ML) corresponds to the half of a lattice constant in the bulk material. The calculations have been performed by using an eight-band $\mathbf{k} \cdot \mathbf{p}$ Hamiltonian [17] with the parameters taken from Ref. [38]. In the Hamiltonian, we also took into account the terms describing the strain effect arising because of the mismatch of lattice constants in the buffer, QW layers, and AlSb barriers. Details of theoretical calculations can be found in Refs. [25,26].

The electronic subbands have been classified as electronlike or holelike levels by comparing the relative contribution to this level at zero quasimomentum from the basis states of $\left|\Gamma_{6}, \pm 1 / 2\right\rangle,\left|\Gamma_{7}, \pm 1 / 2\right\rangle$, and $\left|\Gamma_{8}, \pm 1 / 2\right\rangle$ bands with the contribution from the heavy-hole band $\left|\Gamma_{8}, \pm 3 / 2\right\rangle$ [25]. As the wave functions in $E 1, E 2$ subbands are localized in the InAs layers, while that in the $H 1$ subband is mostly localized in the GaSb layer, the InAs/GaSb/InAs QW can be intuitively considered as a "double QW for electrons" with a GaSb middle barrier, which also plays a role of a "QW for holes" [Fig. 1(a)]. In this case, the $E 1$ and $E 2$ levels can be interpreted as even-odd state splitting, arising from the tunnel-coupled "QWs for electrons." If InAs layers are thin, the $E 1$ subband lies above the holelike subband, and the three-layer QW has a trivial band ordering. Increasing $d_{\text {InAs }}$ induces the mutual inversion between $E 1$ and $H 1$ subbands, resulting in inverted-band structure of the QW. At the critical thickness, the QW hosts single-valley 2D massless Dirac fermions $[39,40]$.

The band gap in the inverted three-layer QWs has a nonmonotonic dependence on the InAs layer thickness. At large $d_{\mathrm{InAs}}$, the gap first becomes indirect and, then, closes due to nonlocal overlapping between conduction and valence bands [17]. As we are interested in a QSHI with the largest possible band gap for the sample grown on $\mathrm{GaSb}$ buffer, we have adjusted the thickness of the InAs and GaSb layers to 34 and $14 \mathrm{MLs}$, respectively. We note that for other buffer material, the maximum gap is achieved at other thickness of InAs and GaSb layers in the QWs [17].

The sample was grown by solid-source molecular beam epitaxy on a semi-insulating (001) GaAs substrate. After deoxidation at around $600{ }^{\circ} \mathrm{C}$ a thick undoped GaAs buffer layer was grown. The large lattice-mismatch between GaAs, $\mathrm{GaSb}$, and AlSb $(\sim 8 \%)$ was accommodated through a thick (more than $1 \mu \mathrm{m}$ ) GaSb buffer layer followed by a ten-period $2.5 \mathrm{~nm} \mathrm{GaSb} / 2.5 \mathrm{~nm}$ AlSb superlattice (SLS), all grown at $500^{\circ} \mathrm{C}$. Subsequently, the substrate temperature was decreased down to $420^{\circ} \mathrm{C}$ to grow the active part of the structures, which consists in three-layer InAs/GaSb/InAs QWs confined by $50-\mathrm{nm}$-thick AlSb barrier layers. A 6-nm GaSb cap layer 
was used to prevent oxidation of the AlSb barrier layers. The shutter sequences at all InAs/GaSb interfaces were organized in order to promote the formation of InSb-like interfaces which gives higher electron mobilities. The sample was nominally undoped. The electron concentration and mobility extracted from magnetotransport measurements at $2 \mathrm{~K}$ were $6.2 \times 10^{11} \mathrm{~cm}^{-2}$ and $2 \times 10^{4} \mathrm{~cm}^{2} / \mathrm{V} \mathrm{s}$, respectively. The band structure and Fermi-level position obtained from the electron concentration are shown in Fig. 1(c).

\section{LANDAU-LEVEL SPECTROSCOPY}

A typical signature of the inverted-band ordering under quantizing magnetic field is the crossing of a particular pair of LLs, which split from the bottom conduction and upper valence subbands and merge under magnetic field $[7,41,42]$. The origin of such a pair, also called zero-mode LLs, becomes clear from an effective Dirac-type Hamiltonian [3] written for the states in $E 1$ and $H 1$ subbands. Below the critical magnetic field $B_{c}$, at which the zero-mode LLs cross, the lowest zero-mode LL has an electronlike character, while the second level, which arises from the conduction band at lower magnetic field $B<B_{c}$, has a heavy-hole-like character, and the band structure remains inverted. At high magnetic field $B>B_{c}$, the system is driven into the trivial band ordering. Finally, in the QWs with trivial band ordering, the electron- and heavy-hole-like level arises at $B=0$ in the conduction and valence band, respectively, and the zero-mode LLs do not cross.

Figure 2(a) provides a LL fan chart for the grown sample. To calculate LL energies and wave functions, we use the axial approximation [25] by keeping the in-plane rotation symmetry. The latter omits the warping terms and the terms resulting from bulk inversion asymmetry of the unit-cell and low-symmetry interfaces [43]. In this case, the wave function for a given LL index $n>0$ generally has eight components, describing the contribution of the $\Gamma_{6}, \Gamma_{7}$, and $\Gamma_{8}$ bands of the bulk material into given LL [44]. Importantly, only seven-, four-, and one-component wave functions are obtained for indices $n=0,-1$, and -2 , respectively. A single, $n=-2$ LL contains only a contribution of the $\left|\Gamma_{8},-3 / 2\right\rangle$ band [25] and its energy decreases nearly linearly with $B$. This level, together with one of the characteristic solutions for $n=0$, represents the zero-mode LLs [27,28,30], discussed above. For comparison, Fig. 2(b) presents a LL fan chart for the QW with trivial band ordering $\left(d_{\mathrm{InAs}}=27 \mathrm{MLs}\right.$ and $\left.d_{\mathrm{GaSb}}=14 \mathrm{MLs}\right)$. As it is seen from Fig. 1(b), the QWs with $d_{\text {InAs }}=34$ MLs and $d_{\text {InAs }}=27$ MLs have comparable band gap at $k=0$ but different band ordering.

The THz magnetotransmission measurements of the grown sample were carried out by using a Fourier transform spectrometer coupled to a 16-T superconducting coil [27,29]. The radiation of a Globar lamp was guided to a sample using oversized waveguides (light pipes). The intensity of the transmitted light was measured by a silicon bolometer. The bolometer was placed in a vacuum chamber separated from the sample chamber. To provide a wide spectral range for the experiments, an indium-sealed cold diamond window ensured the optical coupling between the transmitted light and the bolometer. An additional superconducting coil around the bolometer compensated the spread field of the main coil, keeping the

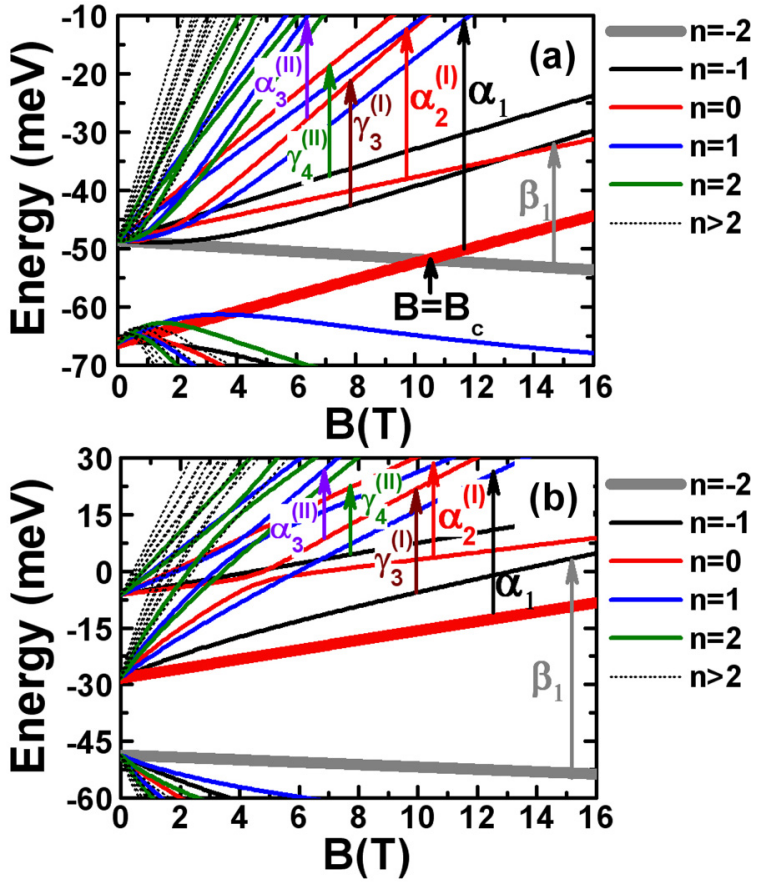

FIG. 2. Landau-level fan chart for the three-layer InAs/GaSb/InAs QW with (a) inverted-band structure, $d_{\mathrm{InAs}}=34$ ML and $d_{\mathrm{GaSb}}=14 \mathrm{ML}$ (the sample under study), and (b) trivial band ordering, $d_{\mathrm{InAs}}=27 \mathrm{ML}$ and $d_{\mathrm{GaSb}}=14 \mathrm{ML}$; also see Fig. 1(b). The numbers in the right side correspond to the LL indices. The brown curve describes the Fermi-level position as a function of magnetic field in the grown sample. The expected dominant absorption transitions are denoted by arrows and Greek letters. A pair of zero-mode LLs with indices -2 and 0 is shown by bold curves.

bolometer at zero magnetic field. This additional compensating superconducting coil also provided an additional screening of the bolometer.

A Lambda plate coil placed below the main magnet allows obtaining superfluid helium around the bolometer and keeping the main coil at $4 \mathrm{~K}$. Thus, our experimental setup allows us to keep the coils in their superconducting state, the bolometer at its optimal temperature, and to tune the sample chamber temperature in the $2-150-\mathrm{K}$ range. The magneto-optical spectra were measured in the Faraday configuration up to $16 \mathrm{~T}$, with a spectral resolution of $0.5 \mathrm{meV}$. All the spectra were normalized by the sample transmission response at $B=0 \mathrm{~T}$.

In the Faraday configuration, optically active inter-LL transitions follow the conventional selection rules $\Delta n= \pm 1$ (for unpolarized radiation) imposed by the electric dipole approximation. However, the proximity of $E 2$ subband and position of the Fermi energy [see Fig. 2(a)] suggest that there are plenty of possible transitions, which satisfy such a rule. Therefore, to identify the absorption lines observed in experimental spectra, we calculate optical matrix elements for transition between the states $l$ and $m$, written as [45]

$$
M=\left(2 m_{0}\right)^{1 / 2}\left\langle\Psi_{m}|\mathbf{e} \cdot \widehat{\mathbf{v}}| \Psi_{l}\right\rangle,
$$

where $m_{0}$ is the free-electron mass, $\Psi_{i}$ is the eight-component envelope function of the eight-band $\mathbf{k} \cdot \mathbf{p}$ Hamiltonian $\hat{H}_{\mathbf{k}}^{8 \times 8}$, $\mathbf{e}$ is the unit vector of light polarization, and $\widehat{\mathbf{v}}$ is the velocity 

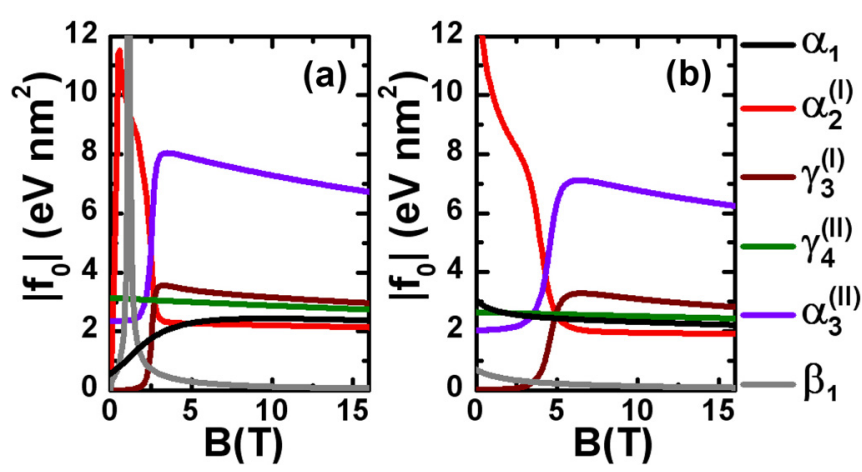

FIG. 3. Oscillator strength $f_{0}=\hbar^{2} \overline{f_{\text {osc }}} /\left(2 m_{0}\right)$ for Landau-level transitions shown in Fig. 2. The overline denotes an averaging over orientation of the light polarization lying in the QW plane. The (a) and (b) panels are connected with those in Fig. 2.

operator, defined by

$$
\hbar \widehat{\mathbf{v}}=\frac{\partial \hat{H}_{\mathbf{k}}^{8 \times 8}}{\partial \widehat{\mathbf{k}}} .
$$

In Eq. (1), $l$ and $m$ are assumed to be the complex symbols, including subband, "spin" indices and LL number $n$ [or quasimomentum $\mathbf{k}_{\|}=\left(k_{x}, k_{y}\right)$ in the QW plane in the absence of magnetic field].

For analysis of the absorption or emission spectra caused by dynamical conductivity [45], instead of optical matrix elements $M$, it is more convenient to operate with the oscillator strength [46] of the given transition defined as

$$
f_{\text {osc }}(m, l)=\frac{2 m_{0}\left|\left\langle\Psi_{m}|\mathbf{e} \cdot \widehat{\mathbf{v}}| \Psi_{l}\right\rangle\right|^{2}}{\left|E_{m}-E_{l}\right|} .
$$

Note that if the orientation of the light polarization projection onto the QW plane is not controlled during performing experiments, one has to perform an averaging of $f_{\text {osc }}(m, l)$ over the orientation of the e component lying in the QW plane.

We have analyzed all possible transitions with $\Delta n= \pm 1$ involving LLs with index $-2,-1,0,1$, which can be observed in the grown sample. Figure 3 shows the oscillator strength for the most intense LL transitions presented in Fig. 2. As expected, the most intense LL transitions are attributed to the cyclotron-resonance (CR)-like transitions in $E 2\left(\alpha_{2}^{(\mathrm{I})}\right.$ and $\gamma_{4}^{(\mathrm{II})}$ transitions) and $H 1\left(\alpha_{3}^{(\mathrm{II})}\right.$ and $\gamma_{3}^{(\mathrm{I})}$ transitions) subbands and to the $\alpha_{1}$ and $\beta_{1}$ transitions from the zero-mode LLs. Other transitions have either vanishing oscillator strength or a strength which is several times lower than the given ones. Note that intersubband LL transitions between $E 2$ and $E 1$ subbands are almost forbidden for all LL indices in the Faraday configuration.

The nonmonotonic behavior of the oscillator strengths in low magnetic fields is caused by numerous anticrossings between LLs with the same indices from $E 2$ and $E 1$ subbands. The latter results in nonmonotonic dependence of the transition energy and optical matrix element $M$ on magnetic field for the given LL transition in the vicinity of the anticrossings. As is clear from Fig. 3, the oscillator strength for the $\alpha_{1}, \alpha_{2}^{(\mathrm{I})}$, $\gamma_{3}^{(\mathrm{I})}, \gamma_{4}^{(\mathrm{II})}, \alpha_{3}^{(\mathrm{II})}$, and $\beta_{1}$ LL transitions in high magnetic fields (a)

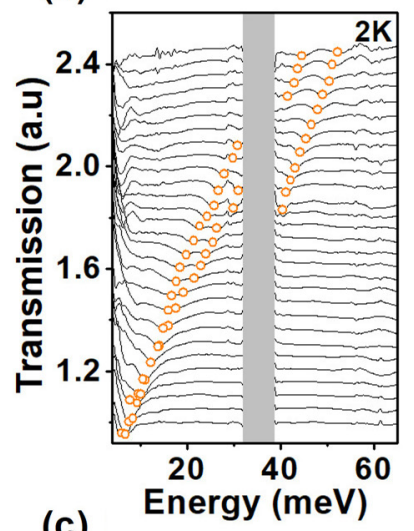

(b)
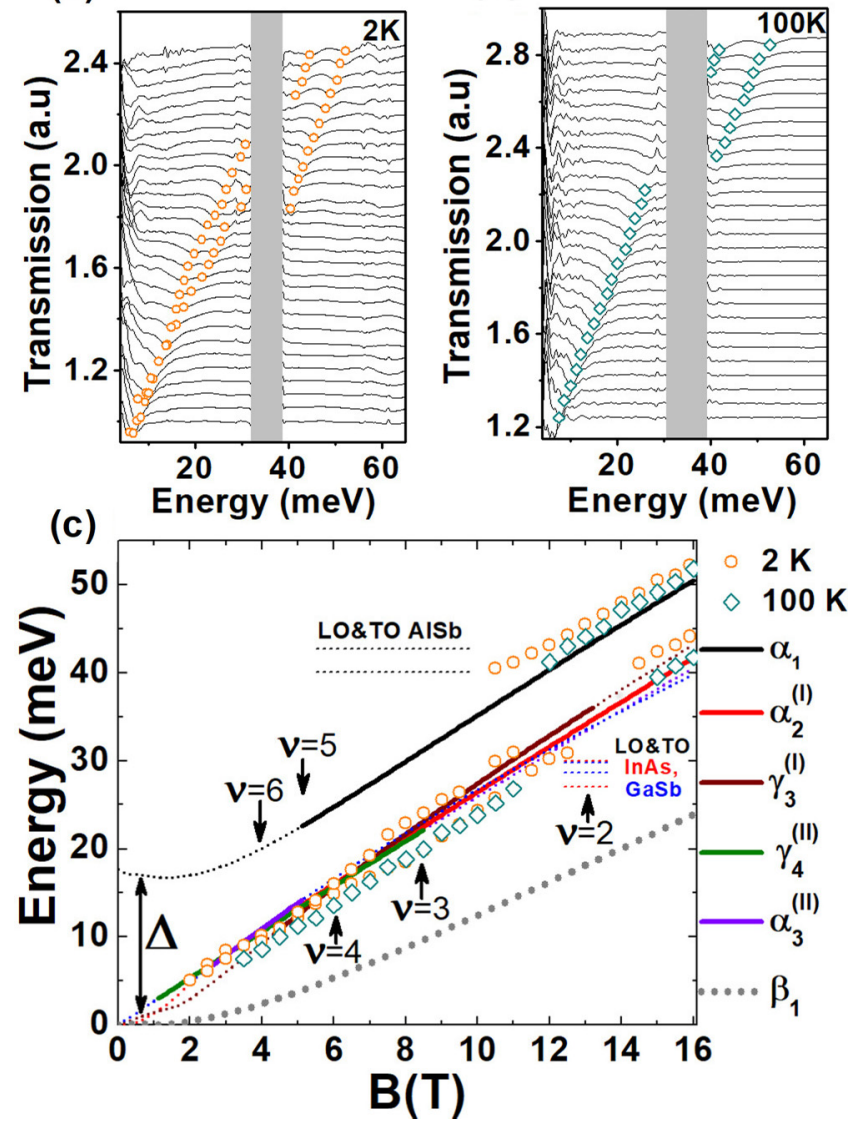

FIG. 4. (a,b) Transmission spectra at 2 and $100 \mathrm{~K}$ from $1 \mathrm{~T}$ (the first plot at the bottom) to $16 \mathrm{~T}$ (the last plot on the top) with a step for the magnetic field of $0.5 \mathrm{~T}$. Shaded areas indicate the Reststrahlen band of GaAs substrate. The symbols mark positions of absorption lines. (c) Fan chart of the most intense LL transitions, marked in Fig. 1(a) by arrows. Experimental data at different temperatures are represented by symbols. The arrows indicate the magnetic fields, corresponding to integer LL filling factors $v$ at $2 \mathrm{~K}$. Horizontal dashed lines correspond to the energies of TO and LO phonons in AlSb (in black, $\omega_{\mathrm{LO}}=42.7 \mathrm{meV}$ and $\omega_{\mathrm{TO}}=40.1 \mathrm{meV}$ ), InAs (in red, $\omega_{\mathrm{LO}}=30 \mathrm{meV}$ and $\omega_{\mathrm{TO}}=27 \mathrm{meV}$ ), and $\mathrm{GaSb}$ (in blue, $\omega_{\mathrm{LO}}=29.8 \mathrm{meV}$ and $\omega_{\mathrm{TO}}=28.6 \mathrm{meV}$ ).

has comparable values and similar behavior for the QWs with trivial and inverted-band structure.

Experimental transmission spectra at 2 and $100 \mathrm{~K}$ are shown in Fig. 4. A comparison of experimental and theoretical transition energies is shown in the bottom panel. The theoretical dependencies, obtained by using band parameters at $2 \mathrm{~K}$ [38], are given by the colored curves. The solid part of each curve represents the range of magnetic fields, in which the LL transition should be observed at electron concentration measured at $2 \mathrm{~K}$. The dotted line for $\beta_{1}$ transition stress the smallest oscillator strength for this transition.

In low magnetic fields, the spectra show a single line attributed to the CR-like transitions in the $E 2$ and $H 1$ subbands. The transitions have close energies due to close values of cyclotron masses $m_{c}$ at the given concentration in the $E 2$ and $H 1$ subbands (see Appendix A). Indeed, the cyclotron mass in the growth at the Fermi level at $2 \mathrm{~K}$ is about $0.0434 m_{0}$ and 
$0.0426 m_{0}$, respectively. Analysis of experimental data gives $m_{c}=(0.044 \pm 0.004) m_{0}$ that is in a good agreement with the theoretical values.

At the energies above the Reststrahlen band, the second line, corresponding to $\alpha_{1}$ transition, arises for both temperatures. It has a linear dependence at high magnetic fields. When the energy tends toward LO phonon energy in AlSb barriers $\left(\omega_{\mathrm{LO}}=\right.$ $40.9 \mathrm{meV}$ ), the energies of the $\alpha_{1}$ and CR-like transitions bend, deviating from the linear behavior. The latter is clearly seen for the measurements at $2 \mathrm{~K}$. We note that this cannot be attributed to electron-phonon interaction because the effect is absent in the vicinity of the phonon energies in InAs $\left(\omega_{\mathrm{LO}}=\right.$ $30 \mathrm{meV}$ and $\left.\omega_{\mathrm{TO}}=27 \mathrm{meV}\right)$ and $\mathrm{GaSb}\left(\omega_{\mathrm{LO}}=29.8 \mathrm{meV}\right.$ and $\omega_{\mathrm{TO}}=28.6 \mathrm{meV}$ ) layers.

The observed energy bending has a pure optical origin. In our case, the optical response of the system is governed by two strongly resonant processes: the resonant dielectric function of the material at the phonon's (both TO and LO) frequencies (even if the polaron effect is small) and magnetoabsorption of the $2 \mathrm{D}$ electron system. Thus, the profile of absorption lines can be strongly modified by the singularities of the dielectric function at a phonon's frequencies of the QW and barrier materials. As the symbols in Fig. 4 define the maxima of the resulting absorption line, the latter produces the bending effect of the resonant energy in the vicinity of $\omega_{\mathrm{TO}}$ or $\omega_{\mathrm{LO}}$ [47].

As it is seen from Fig. 4(c), experimental data at both temperatures are qualitatively in good agreement with theoretical prediction. The splitting of absorption line arising at $2 \mathrm{~K}$ in the spectra at $B=7-11 \mathrm{~T}$ can be interpreted as a difference in the energies of CR-like transitions in the $E 2$ and $H 1$ subbands, respectively. Interestingly, increasing the temperature does not change the positions of the absorption lines at $B=7-11 \mathrm{~T}$ corresponding to the low-energy peaks, while the high-energy component of the split lines disappears completely at $100 \mathrm{~K}$. The latter is directly caused by a change of the electron concentration with temperature, which affects the filling factor of the LLs. A larger experimental splitting of the absorption lines may result from the effects of bulk inversion asymmetry and low-symmetry interfaces [43] ignored in our calculations. Notably, the effect of electron-electron interaction may also influence close energies of LL transitions [33] as we deal with strongly nonparabolic 2D systems for which Kohn's theorem does not hold.

The LL transition energies are independent of temperature, as clearly seen in high magnetic fields at the energies above the Reststrahlen band. Although the position of the low-energy line, which at various concentrations can be attributed to different CR-like transitions, is slightly changing with temperature, the position of the high-energy line, identified as the $\alpha_{1}$ interband LL transition, remains the same at 2 and $100 \mathrm{~K}$. It also indicates that the resonant energy caused by the given LL transition is temperature independent.

The main difference of our sample from the inverted $\mathrm{HgTe}$ QWs $[27,30,31,34]$ is that the line related with the $\beta_{1}$ transition, involving the second zero-mode LL, is not observed (see $B>13 \mathrm{~T}$ that correspond to $v<2$ ) due to small values of the oscillator strength (see Fig. 3). The latter can be used to discriminate the inverted and trivial band ordering in the InAs/GaSb/InAs QWs by LL spectroscopy. As it is seen from
Fig. 2, if the band structure is inverted, the $\alpha_{1}$ transition has the highest energy in high magnetic fields that leans towards the gap energy at $k=0$ when $B$ tends to zero. For trivial band ordering, the same behavior is relevant but for the $\beta_{1}$ transition (see Appendix B). As the oscillator strengths weakly depend on the band ordering (see Fig. 3), one also should not observe the interband $\beta_{1}$ LL transition in the QW with trivial band ordering. Since other LL transitions in the trivial QW are CR-like, observation of the high-energy line corresponding to the interband LL transition evidences the inverted-band structure of our sample.

Unfortunately, large electron concentration in our samples makes impossible to follow the energy of the $\alpha_{1}$ transition down to low magnetic fields. Therefore, we cannot directly extract the gap at $k=0$ from magnetospectroscopy. However, we can estimate the difference in energies for CR-like and $\alpha_{1}$ transitions, marked as $\Delta$. The value of $\Delta$ at $B=0$ defines the gap. Although it decreases with magnetic field, $\Delta$ tends to a constant value at the high fields. From our experimental data, we have found $\Delta \approx 8 \mathrm{meV}$ for $B>10 \mathrm{~T}$.

\section{THZ PHOTOLUMINESCENCE}

The energy gaps between $E 2, E 1$, and $H 1$ subbands at $B=0$ can be obtained by means of THz PL spectroscopy. In the case of inverted three-layer InAs/GaSb QWs, one may observe both interband $\mathrm{PL}$ between conduction and valence subbands (H1-E1, E2-E1) and intersubband PL between conduction subbands $(E 2-H 1)$. The intersubband PL is possible because the energy difference between subbands is well below the energy of optical phonons in InAs and GaSb. In the interband $\mathrm{PL}$, an electron from the valence subband is excited into the conduction subband, leaving behind a hole. The electron and hole then relax independently, through electron-electron and electron-phonon interactions, toward the Fermi level and the top of valence subband, respectively. Reaching the subband edge, the nonthermalized hole can recombine either radiatively or nonradiatively with the electron from the bottom of conduction subband.

The PL spectroscopy have been performed in a closedcycle cryostat optically connected to a Fourier transform spectrometer, operating in the step scan mode. The temperature in the cryostat was varied in the range from 20 to $50 \mathrm{~K}$. For PL pumping, a continuous-wave Ti:sapphire laser operating at $800 \mathrm{~nm}$ wavelength has been used. The laser beam was mechanically chopped with modulation frequency lying in the $100-400-\mathrm{Hz}$ range. Excitation power was varied from 10 to $300 \mathrm{~mW}$. The beam spot at the sample was about 3 $\mathrm{mm}$ in diameter. The samples have been excited from the top of the structure by $45^{\circ}$-tilted incident pumping radiation. The PL radiation was collected from $45^{\circ}$ direction relative to growth axis. Detection was performed with $4.2-\mathrm{K}$ cooled silicon bolometer and lock-in detection technique was used to extract the luminescence signal from that of the black-body radiation [36,37]. The PL spectra for the temperatures of 20 and $50 \mathrm{~K}$ measured at $300-\mathrm{mW}$ excitation power are presented in Fig. 5. The insets show the oscillator strength of optical transitions between $E 2, E 1$, and $H 1$ subbands as a function of quasimomentum oriented along (110) direction. 


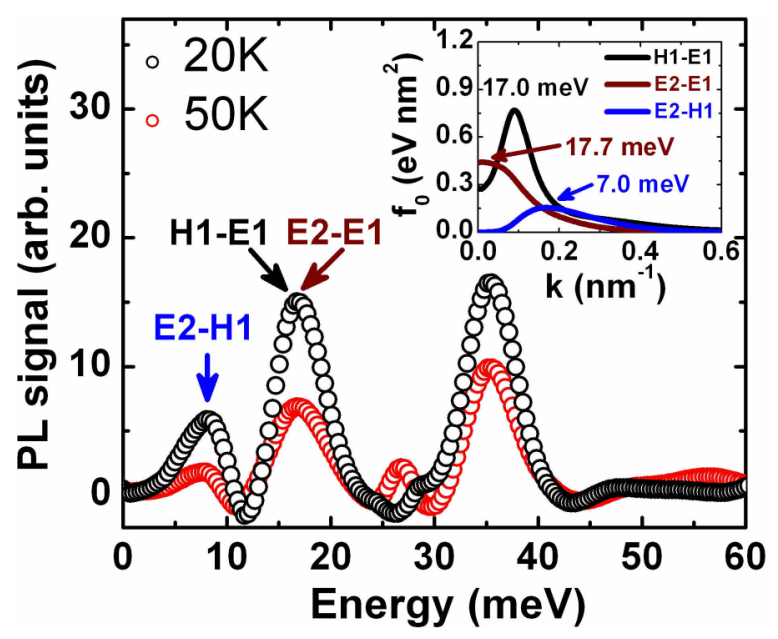

FIG. 5. THz photoluminescence spectra at different temperatures measured from $45^{\circ}$ direction relative to growth axis. The insets show oscillator strength $f_{0}=\hbar^{2} \overline{f_{\text {osc }}} /\left(2 m_{0}\right)$ for optical transitions between $E 2, E 1$, and $H 1$ subbands calculated for the experimental conditions. The overline denotes an averaging over orientation of the polarization component lying in the QW plane. The numbers correspond to the transition energies at maxima of $f_{0}$.

The spectra obtained under oblique pumping radiation show three well-resolved emission lines. The high-energy line observed at about $35 \mathrm{meV}$ corresponds to the luminescence of the $\mathrm{Ga}$ antisite defects from the GaSb buffer [48-50]. The lowenergy line at $7.5 \mathrm{meV}$ may be related to the $E 2-H 1$ transition. Its position is in good agreement with the energy corresponding to the maximum value of the oscillator strength. The middle line at $16.5 \mathrm{meV}$ is a superposition of $H 1-E 1$ and $E 2-E 1$ transitions, which have close energies. Indeed, the energy of maximum of the oscillator strength for these transitions differs by less than $1 \mathrm{meV}$. Therefore, we cannot resolve them in the PL spectra. We note that the observed $E 2-H 1$ and $E 2-E 1$ transitions are active in $z$ polarization, while the $E 1-H 1$ transition is mostly active in the polarization parallel to the $\mathrm{QW}$ plane. Figure 5 also shows that increasing temperature up to $50 \mathrm{~K}$ does not change the position of the emission lines for the transitions between $E 2, E 1$, and $H 1$ subbands. This means that the gaps between $E 2$, $E 1$, and $H 1$ subbands are independent of temperature, which is consistent with the results obtained by temperature-dependent LL spectroscopy.

\section{SUMMARY AND PERSPECTIVES}

Figure 6 summarizes our results. It provides a comparison between the experimental values and the theoretical calculations of $\Delta$ defined as a difference in energies of the $\alpha_{1}$ and $\gamma_{3}^{(\mathrm{I})}$ transitions (see Figs. 2 and 4). As seen from Fig. 1(c), the sample has a loop of local maxima in the valence subband in the vicinity of $k=0$, and the band gap in our sample is indirect with a value of about $15.5 \mathrm{meV}$. Our experimental value of $\Delta \simeq 17 \mathrm{meV}$, corresponding to the optical gap in the vicinity of the $\Gamma$ point, is very close to the indirect band gap. Figure 6 shows a good agreement between the theoretical and experimental values of $\Delta$.

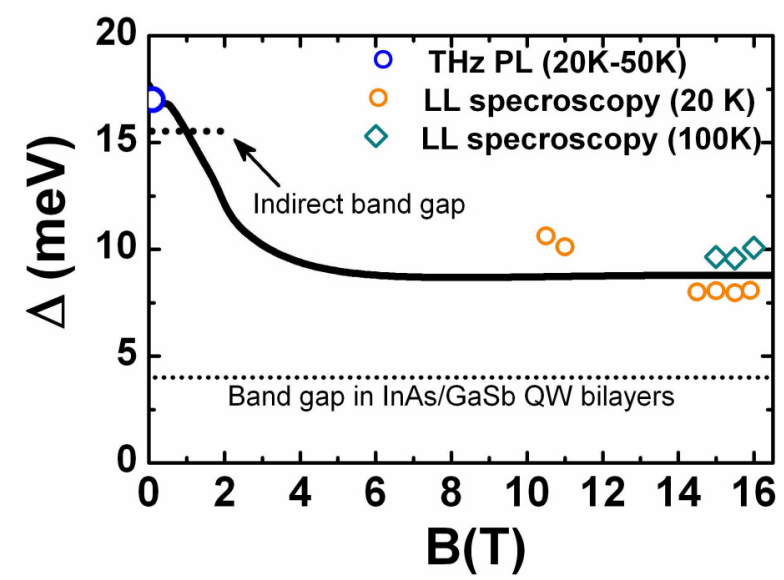

FIG. 6. Dependence of $\Delta$ on magnetic field. The solid line represents theoretical dependence extracted from LL fan chart. The symbols show the experimental values, obtained from LL magnetospectroscopy and PL. The dotted lines mark the band gap in our sample and the maximum inverted gap of InAs/GaSb QW bilayers.

In conclusion, we have performed temperature-dependent $\mathrm{THz}$ spectroscopy of inverted three-layer InAs/GaSb/InAs QW. By performing LL spectroscopy, we have observed CRlike transitions in the two lowest conduction subbands, as well as the interband LL transition from one of the zero-mode LLs in magnetic fields up to $16 \mathrm{~T}$. Moreover, we argue that the observed difference in energies between CR-like transition and transition from the zero-mode LL is directly related with inverted-band structure of our sample. The transition energies have been found to be independent of temperature in the range up to $100 \mathrm{~K}$. To probe the optical gap in zero magnetic field, we have also performed the measurements of THz PL. We have demonstrated that positions of the observed emission lines corresponding to optical transitions between $E 2, E 1$, and $H 1$ subbands are also independent of temperature and in good agreement with the resonant energies corresponding to the maximum of the oscillator strengths.

Although the Fermi level lies in the conduction band in our sample and the existence of QSHI state in the three-layer QWs has experimentally yet to be established by a direct transport measurement of the quantum spin Hall effect with its universal quantized conductance, the agreement between experimental results and theoretical prediction already strongly suggests that QSHI state can be indeed realized in these structures. Direct measurements of the optical gap have revealed a value of about $17 \mathrm{meV}$, which is not only four times larger than the maximum band gap available in the inverted InAs/GaSb QW bilayers but comparable with the nontrivial gap in $\mathrm{HgTe} / \mathrm{CdHgTe} \mathrm{QWs}$ grown on CdTe buffer. However, in contrast to the HgTe QWs [24-28], our experimental results show that the invertedband gap in three-layer InAs/GaSb/InAs QWs is temperature independent.

\section{ACKNOWLEDGMENTS}

Temperature-dependent magnetospectroscopy was supported by MIPS department of Montpellier University through the "Occitanie Terahertz Platform," by the LanguedocRoussillon region via the "Gepeto Terahertz platform" and 

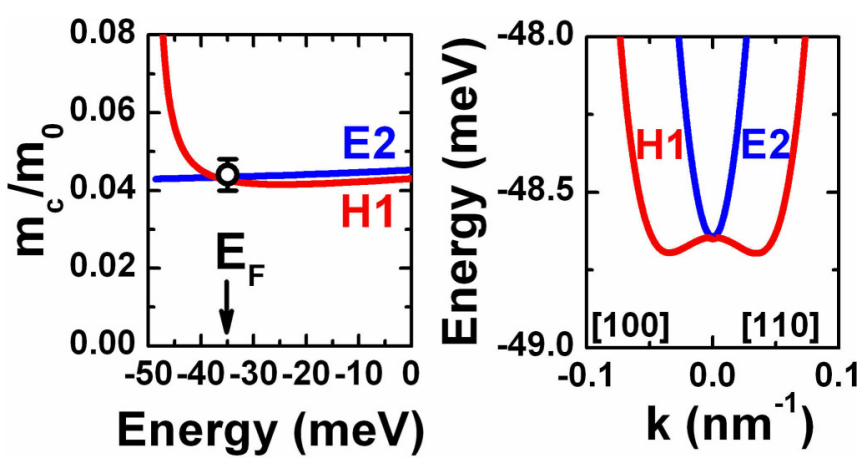

FIG. 7. Cyclotron mass $m_{c}$ in conduction subbands as a function of energy in both samples. The open symbols correspond to the experimental values of cyclotron mass extracted from analysis of the absorption lines corresponding to CR-like transitions in low magnetic fields. The right panel shows dispersion of conduction subbands in the vicinity of $k=0$.

the ARPE project "Terasens," and by the CNRS through "Emergence project 2016" and LIA "TeraMIR." MBE growth of the samples was performed within the French program on "Investments for the Future" (Grant No. ANR-11-EQPX0016). Temperature-dependent PL spectroscopy and theoretical support were performed in the framework of Project No. 17-72-10158 provided by the Russian Science Foundation. M.A.F. and A.M.K. acknowledge the Russian Ministry of Education and Science of the Russian Federation (Grant No. MK-1136.2017.2) and Program No. 32 of RAS.

\section{APPENDIX A: QUASICLASSICAL CYCLOTRON RESONANCE}

Figure 7 provides dependence of cyclotron mass on energy in conduction subbands for the grown sample. Experimental value extracted from position of the absorption line corresponding to CR-like transitions in low magnetic fields is presented by the symbol. The cyclotron mass has been calculated by applying a quasiclassical quantization rule, which leads to

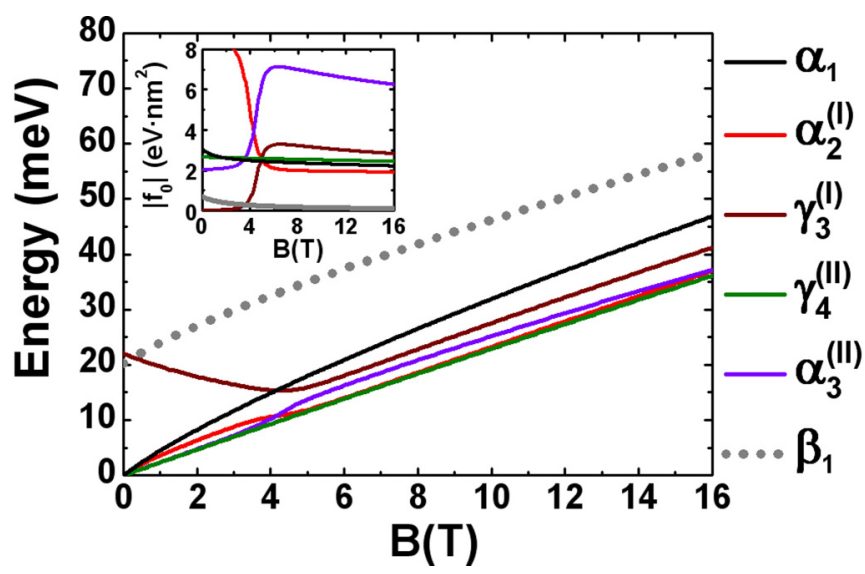

FIG. 8. Fan chart of the most intense LL transitions in the threelayer InAs/GaSb/InAs QW with trivial band ordering, $d_{\mathrm{InAs}}=27 \mathrm{ML}$ and $d_{\mathrm{GaSb}}=14 \mathrm{ML}$. The oscillator strength $f_{0}$ for the transitions are shown in the inset.

the following expression for the cyclotron mass:

$$
m_{c}=\frac{\hbar^{2}}{2 \pi} \frac{d}{d E} S_{i}(E)
$$

where $S_{i}(E)$ is the area of the single closed contour in $k$ space at energy $E$ in the subband with index $i$. The right panel in Fig. 7 shows the "W"-like shape of the $H 1$ subband, which is not clear in Fig. 1(c). The latter results in nonmonotonic behavior of $\alpha_{1}$ LL transition as a function of magnetic field.

\section{APPENDIX B: LANDAU-LEVEL TRANSITIONS FOR TRIVIAL BAND ORDERING}

Figure 8 presents a fan chart for the most intense LL transitions for the $\mathrm{QW}$ with trivial band ordering at $d_{\mathrm{InAs}}=27$ ML and $d_{\mathrm{GaSb}}=14 \mathrm{ML}$ [also see Fig. 2(b)]. As it is mentioned in the main text, the interband $\beta_{1}$ LL transition should be observed due to the small oscillator strength. Therefore, in contrast to the QW with inverted-band ordering, only CR-like transitions should arise in the magneto-optical spectra of the trivial QWs.
[1] C. L. Kane and E. J. Mele, Phys. Rev. Lett. 95, 226801 (2005).

[2] B. A. Bernevig and S.-C. Zhang, Phys. Rev. Lett. 96, 106802 (2006).

[3] B. A. Bernevig, T. L. Hughes, and S.-C. Zhang, Science 314, 1757 (2006).

[4] C. Liu, T. L. Hughes, X.-L. Qi, K. Wang, and S.-C. Zhang, Phys. Rev. Lett. 100, 236601 (2008).

[5] M. Z. Hasan and C. L. Kane, Rev. Mod. Phys. 82, 3045 (2010).

[6] X.-L. Qi and S.-C. Zhang, Rev. Mod. Phys. 83, 1057 (2011).

[7] M. König, S. Wiedmann, C. Brüne, A. Roth, H. Buhmann, L. W. Molenkamp, X.-L. Qi, and S.-C. Zhang, Science 318, 766 (2007).

[8] I. Knez, R.-R. Du, and G. Sullivan, Phys. Rev. Lett. 107, 136603 (2011).

[9] L. G. Gerchikov and A. V. Subashiev, Phys. Status Solidi B 160, 443 (1990).
[10] P. Leubner, L. Lunczer, C. Brüne, H. Buhmann, and L. W. Molenkamp, Phys. Rev. Lett. 117, 086403 (2016).

[11] Y. Naveh and B. Laikhtman, Appl. Phys. Lett. 66, 1980 (1995).

[12] M. J. Yang, C. H. Yang, B. R. Bennett, and B. V. Shanabrook, Phys. Rev. Lett. 78, 4613 (1997).

[13] M. Drndic, M. P. Grimshaw, L. J. Cooper, D. A. Ritchie, and N. K. Patel, Appl. Phys. Lett. 70, 481 (1997).

[14] S. Murakami, S. Iso, Y. Avishai, M. Onoda, and N. Nagaosa, Phys. Rev. B 76, 205304 (2007).

[15] T. Akiho, F. Couedo, H. Irie, K. Suzuki, K. Onomitsu, and K. Muraki, Appl. Phys. Lett. 109, 192105 (2016).

[16] L. Du, T. Li, W. Lou, X. Wu, X. Liu, Z. Han, C. Zhang, G. Sullivan, A. Ikhlassi, K. Chang, and R.-R. Du, Phys. Rev. Lett. 119, 056803 (2017).

[17] S. S. Krishtopenko and F. Teppe, Sci. Adv. 4, eaap7529 (2018).

[18] S. Wu, V. Fatemi, Q. D. Gibson, K. Watanabe, T. Taniguchi, R. J. Cava, and P. Jarillo-Herrero, Science 359, 76 (2018). 
[19] Y. Cao, A. Mishchenko, G. L. Yu, E. Khestanova, A. P. Rooney, E. Prestat, A. V. Kretinin, P. Blake, M. B. Shalom, C. Woods, J. Chapman, G. Balakrishnan, I. V. Grigorieva, K. S. Novoselov, B. A. Piot, M. Potemski, K. Watanabe, T. Taniguchi, S. J. Haigh, A. K. Geim, and R. V. Gorbachev, Nano Lett. 15, 4914 (2015).

[20] L. Wang, I. Gutierrez-Lezama, C. Barreteau, N. Ubrig, E. Giannini, and A. F. Morpurgo, Nat. Commun. 6, 8892 (2015).

[21] F. Ye, J. Lee, J. Hu, Z. Mao, J. Wei, and P. X.-L. Feng, Small 12, 5802 (2016).

[22] Z. Fei, T. Palomaki, S. Wu, W. Zhao, X. Cai, B. Sun, P. Nguyen, J. Finney, X. Xu, and D. H. Cobden, Nat. Phys. 13, 677 (2017).

[23] S. Tang, C. Zhang, D. Wong, Z. Pedramrazi, H.-Z. Tsai, C. Jia, B. Moritz, M. Claassen, H. Ryu, S. Kahn, J. Jiang, H. Yan, M. Hashimoto, D. Lu, R. G. Moore, C.-C. Hwang, C. Hwang, Z. Hussain, Y. Chen, M. M. Ugeda et al., Nat. Phys. 13, 683 (2017).

[24] S. Wiedmann, A. Jost, C. Thienel, C. Brüne, P. Leubner, H. Buhmann, L. W. Molenkamp, J. C. Maan, and U. Zeitler, Phys. Rev. B 91, 205311 (2015).

[25] S. S. Krishtopenko, I. Yahniuk, D. B. But, V. I. Gavrilenko, W. Knap, and F. Teppe, Phys. Rev. B 94, 245402 (2016).

[26] S. S. Krishtopenko, W. Knap, and F. Teppe, Sci. Rep. 6, 30755 (2016).

[27] M. Marcinkiewicz, S. Ruffenach, S. S. Krishtopenko, A. M. Kadykov, C. Consejo, D. B. But, W. Desrat, W. Knap, J. Torres, A. V. Ikonnikov, K. E. Spirin, S. V. Morozov, V. I. Gavrilenko, N. N. Mikhailov, S. A. Dvoretskii, and F. Teppe, Phys. Rev. B 96, 035405 (2017).

[28] A. M. Kadykov, S. S. Krishtopenko, B. Jouault, W. Desrat, W. Knap, S. Ruffenach, C. Consejo, J. Torres, S. V. Morozov, N. N. Mikhailov, S. A. Dvoretskii, and F. Teppe, Phys. Rev. Lett. 120, 086401 (2018).

[29] F. Teppe, M. Marcinkiewicz, S. S. Krishtopenko, S. Ruffenach, C. Consejo, A. M. Kadykov, W. Desrat, D. But, W. Knap, J. Ludwig, S. Moon, D. Smirnov, M. Orlita, Z. Jiang, S. V. Morozov, V. Gavrilenko, N. N. Mikhailov, and S. A. Dvoretskii, Nat. Commun. 7, 12576 (2016).

[30] M. Orlita, K. Masztalerz, C. Faugeras, M. Potemski, E. G. Novik, C. Brüne, H. Buhmann, and L. W. Molenkamp, Phys. Rev. B 83, 115307 (2011).

[31] M. Zholudev, F. Teppe, M. Orlita, C. Consejo, J. Torres, N. Dyakonova, M. Czapkiewicz, J. Wróbel, G. Grabecki, N. Mikhailov, S. Dvoretskii, A. Ikonnikov, K. Spirin, V. Aleshkin, V. Gavrilenko, and W. Knap, Phys. Rev. B 86, 205420 (2012).

[32] S. S. Krishtopenko, A. V. Ikonnikov, A. V. Maremyanin, K. E. Spirin, V. I. Gavrilenko, Y. G. Sadofyev, M. Goiran, M. Sadowsky, and Y. B. Vasilyev, J. Appl. Phys. 111, 093711 (2012).

[33] S. S. Krishtopenko, A. V. Ikonnikov, M. Orlita, Y. G. Sadofyev, M. Goiran, F. Teppe, W. Knap, and V. I. Gavrilenko, J. Appl. Phys. 117, 112813 (2015).
[34] A. V. Ikonnikov, S. S. Krishtopenko, O. Drachenko, M. Goiran, M. S. Zholudev, V. V. Platonov, Y. B. Kudasov, A. S. Korshunov, D. A. Maslov, I. V. Makarov, O. M. Surdin, A. V. Philippov, M. Marcinkiewicz, S. Ruffenach, F. Teppe, W. Knap, N. N. Mikhailov, S. A. Dvoretsky, and V. I. Gavrilenko, Phys. Rev. B 94, 155421 (2016).

[35] B. A. Assaf, T. Phuphachong, V. V. Volobuev, A. Inhofer, G. Bauer, G. Springholz, L. A. de Vaulchier, and Y. Guldner, Sci. Rep. 6, 20323 (2016).

[36] S. V. Morozov, V. V. Rumyantsev, A. V. Antonov, K. V. Maremyanin, K. E. Kudryavtsev, L. V. Krasilnikova, N. N. Mikhailov, S. A. Dvoretskii, and V. I. Gavrilenko, Appl. Phys. Lett. 104, 072102 (2014).

[37] S. V. Morozov, V. V. Rumyantsev, A. V. Antonov, A. M. Kadykov, K. V. Maremyanin, K. E. Kudryavtsev, N. N Mikhailov, S. A. Dvoretskii, and V. I. Gavrilenko, Appl. Phys. Lett. 105, 022102 (2014).

[38] I. Vurgaftman, J. R. Meyer, and L. R. Ram-Mohan, J. Appl. Phys. 89, 5815 (2001).

[39] S. Ruffenach, S. S. Krishtopenko, L. S. Bovkun, A. V. Ikonnikov, M. Marcinkiewicz, C. Consejo, M. Potemski, B. Piot, M. Orlita, B. R. Semyagin, M. A. Putyato, E. A. Emelyanov, V. V. Preobrazhenskii, W. Knap, F. Gonzalez-Posada, G. Boissier, E. Tournié, F. Teppe, and V. I. Gavrilenko, JETP Lett. 106, 727 (2017).

[40] S. S. Krishtopenko, A. V. Ikonnikov, K. V. Maremyanin, L. S. Bovkun, K. E. Spirin, A. M. Kadykov, M. Marcinkiewicz, S. Ruffenach, C. Consejo, F. Teppe, W. Knap, B. R. Semyagin, M. A. Putyato, E. A. Emelyanov, V. V. Preobrazhenskii, and V. I. Gavrilenko, Semiconductors 51, 38 (2017).

[41] S.-B. Zhang, Y.-Y. Zhang, and S.-Q. Shen, Phys. Rev. B 90, 115305 (2014).

[42] Y. Jiang, S. Thapa, G. D. Sanders, C. J. Stanton, Q. Zhang, J. Kono, W. K. Lou, K. Chang, S. D. Hawkins, J. F. Klem, W. Pan, D. Smirnov, and Z. Jiang, Phys. Rev. B 95, 045116 (2017).

[43] I. Semenikhin, A. Zakharova, K. Nilsson, and K. A. Chao, Phys. Rev. B 76, 035335 (2007).

[44] S. S. Krishtopenko, V. I. Gavrilenko, and M. Goiran, Phys. Rev. B 87, 155113 (2013).

[45] R. Winkler, M. Merkler, T. Darnhofer, and U. Rössler, Phys. Rev. B 53, 10858 (1996).

[46] C. H. Henry and K. Nassau, Phys. Rev. B 1, 1628 (1970).

[47] M. Ziesmann, D. Heitmann, and L. L. Chang, Phys. Rev. B 35, 4541 (1987).

[48] C. C. Ling, M. K. Lui, S. K. Ma, X. D. Chen, S. Fung, and C. D. Beling, Appl. Phys. Lett. 85, 384 (2004).

[49] J. Kujala, N. Segercrantz, F. Tuomisto, and J. Slotte, J. Appl. Phys. 116, 143508 (2014).

[50] N. Segercrantz, J. Slotte, I. Makkonen, J. Kujala, F. Tuomisto, Y. Song, and S. Wang, Appl. Phys. Lett. 105, 082113 (2014). 